BNL - 66994

\title{
INVESTIGATION OF COHERENT EMISSION FROM THE NSLS VUV RING
}

\author{
G.L. Carr, S.L. Kramer and J.B. Murphy \\ Brookhaven National Laboratory \\ Upton, New York 11973 \\ J. LaVeigne, R.P.S.M. Lobo, D.H. Reitze and D.B. Tanner \\ University of Florida \\ Gainesville, Florida 32611
}

March 1999

\section{National Synchrotron Light Source}

\author{
Brookhaven National Laboratory \\ Operated by \\ Brookhaven Science Associates \\ Upton, NY 11973
}

Under Contract with the United States Department of Energy

Contract Number DE-AC02-98CH10886 


\section{DISCLAIMER}

This report was prepared as an account of work sponsored by an agency of the United States Government. Neither the United States Government nor any agency thereof, nor any of their employees, nor any of their contractors, subcontractors or their employees, makes any warranty, express or implied, or assumes any legal liability or responsibility for the accuracy, completeness, or any third party's use or the results of such use of any information, apparatus, product, or process disclosed, or represents that its use would not infringe privately owned rights. Reference herein to any specific commercial product, process, or service by trade name, trademark, manufacturer, or otherwise, does not necessarily constitute or imply its endorsement, recommendation, or favoring by the United States Government or any agency thereof or its contractors or subcontractors. The views and opinions of authors expressed herein do not necessarily state or reflect those of the United States Government or any agency thereof.

\section{Investigation of Coherent Emission from the NSLS VUV Ring}




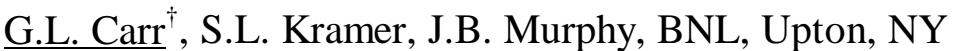 \\ J. LaVeigne, R.P.S.M. Lobo, D.H. Reitze, D.B. Tanner, Univ. Florida, Gainesville, FL}

\begin{abstract}
Bursts of coherent radiation are observed from the NSLS VUV ring near a wavelength of $7 \mathrm{~mm}$. The bursts occur when the electron beam current (I) exceeds a threshold value $\left(\mathrm{I}_{\mathrm{th}}\right)$, which itself varies with ring operating conditions. Beyond threshold, the average intensity of the emission is found to increase as $\left(\mathrm{I}-\mathrm{I}_{\mathrm{th}}\right)^{2}$. With other parameters held nearly constant, the threshold current value is found to increase quadratically with synchrotron frequency, indicating a linear dependence on momentum compaction. It is believed that the coherent emission is a consequence of micro-bunching of the electron beam due to the microwave instability.
\end{abstract}

\section{INTRODUCTION}

Synchrotron radiation is produced when electron bunches traverse the magnetic guide structure of an accelerator or storage ring. For wavelengths shorter than the bunch length, the incoherent superposition of each electron's radiated field results in an intensity that scales linearly with the number of electrons, N. For wavelengths longer than the bunch, the resulting superposition has a large coherent term which scales as $\mathrm{N}^{2}$. The spectral content of this coherent emission is the product of the standard synchrotron radiation flux and the Fourier transform of the longitudinal electron density[1,2]. Thus, for a Gaussian bunch of width $\sigma_{\mathrm{L}}$, the spectral range for coherent emission is also Gaussian with width $\sigma_{\omega}=\mathrm{c} / 2 \pi \sigma_{\mathrm{L}}$. A $10 \mathrm{~cm}$ electron bunch (300 ps duration) emits coherently for frequencies up to $\sim 1 \mathrm{GHz}$. Such emission is difficult to observe since the radiation must propagate out from the ring chamber to a point where a detector is situated. The ring chamber is a waveguide[1], and the dimensions of most chambers are such that the effective cutoff frequency is above $10 \mathrm{GHz}$. However, if the electron bunch is small enough (i.e., $\sim 10 \mathrm{ps}$ duration) or a short period modulation of the bunch density can be imposed, coherent emission at higher frequencies should be observed.

In addition to intentional modifications of the electron bunch, instabilities can lead to variations in the bunch shape and density, which in turn may lead to coherent emission. This work presents evidence for the emission of coherent synchrotron radiation from the NSLS VUV

\footnotetext{
"Work supported by the U.S. Dept. of Energy through contracts DE-AC0298CH10886 at the NSLS and DE-FG02-96ER45584 at Florida.

$†$ Email: carr@bnl.gov
}

ring. The radiation occurs in bursts of less than $1 \mathrm{~ms}$ in duration at a wavelength of $7 \mathrm{~mm}$; much shorter than the nominal electron bunch length. This and other evidence suggest that the emission is a consequence of microbunching (a density modulation within a bunch) that is characteristic of the microwave instability.

\section{EXPERIMENT}

\subsection{NSLS VUV Ring}

Our measurements were conducted using the VUV ring at the National Synchrotron Light Source (NSLS/BNL). This storage ring operates as a dedicated synchrotron radiation source. It has a Chasman-Green lattice consisting of 4 superperiods. The bending radius is $1.91 \mathrm{~m}$ in each of the 8 dipole magnets. The rf accelerating system operates at $52.88 \mathrm{MHz}$ and the ring orbit frequency is $5.7 \mathrm{MHz}$. Though the ring energy can be varied from below $500 \mathrm{MeV}$ up to $800 \mathrm{MeV}$, we conducted most of our studies at the injection energy of $737 \mathrm{MeV}$. In the standard mode of operation, the synchrotron tune is near $11.8 \mathrm{kHz}$, corresponding to a momentum compaction $\alpha=0.0235$. By varying $\alpha$, the synchrotron tune can be changed by more than an order of magnitude, and $\alpha$ can be set to both positive and negative values.

\subsection{U12IR beamline}

The presence of short wavelength coherent emission is detected at U12IR, one of 6 infrared beamlines at the NSLS built to extract infrared radiation from a bending magnet port. The U12IR beamline extracts $90 \mathrm{mr}$ by $90 \mathrm{mr}$ through a $6 \mathrm{~cm}$ square aperture, which is somewhat larger than the interior dimension of the ring chamber itself. Infrared light is transported by both mirror and waveguide optics to a lamellar grating interferometer and $\ell \mathrm{He}$ cooled bolometric detector. The longest detectable wavelength for this system is $\sim 20 \mathrm{~mm}$. The detector response has a fall time between 200 and $800 \mu \mathrm{s}$. The rise time is less well known, but is substantially shorter (a few microseconds).

\subsection{Coherent bursts}

Under normal operations of the VUV ring, the far IR power is temporally smooth and varies linearly with beam current, as expected for incoherent synchrotron radiation. When the beam current exceeds a threshold value (which depends on the ring's operating parameters), bursts of radiation are emitted. An example of these bursts is shown in Figure 1. The time structure is 
rather complex, and varies with operating conditions. Typically, bursts occur with varying amplitude, and at time intervals ranging from $\sim 1 \mathrm{~ms}$ to $\sim 10 \mathrm{~ms}$. The growth rate of these particular bursts is faster than the resolution of our detector (a few microseconds). The decay time is also detector limited at $\sim 200 \mu \mathrm{s}$. As the current is increased beyond the threshold value, the magnitude of a typical burst increases. Figure 2 shows that the time-average power increases as the square of the excess beam current (i.e., current over threshold).

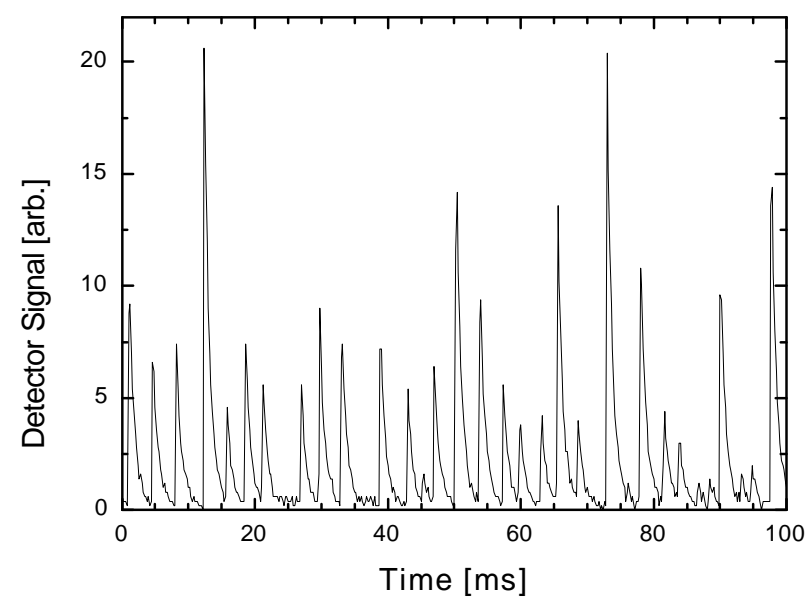

Figure 1: Far infrared detector output versus time showing emission bursts for $\mathrm{I}>\mathrm{I}_{\mathrm{th}}$.

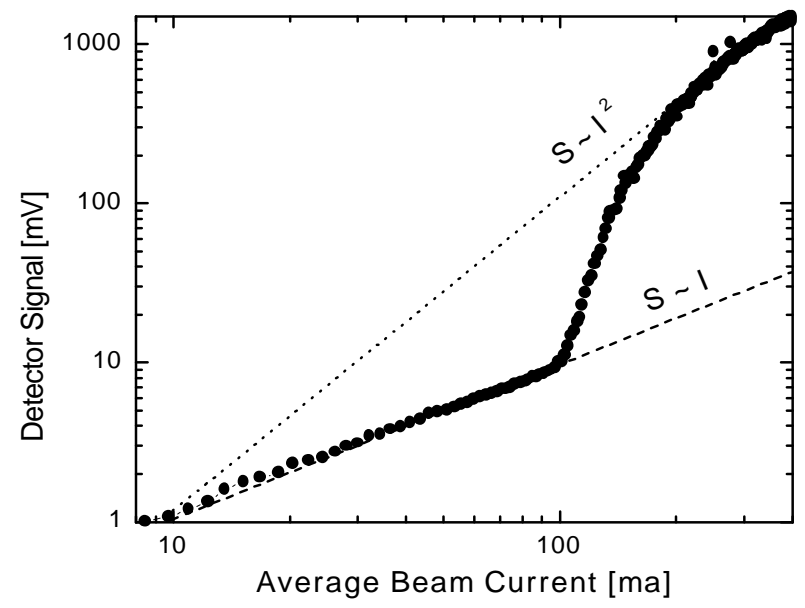

Figure 2: Measured output power versus average beam current in a single bunch, showing the onset of coherent emission at $\mathrm{I}_{\mathrm{th}}=100 \mathrm{ma}$.

We measured the coherent emission spectral content using a lamellar grating interferometer, which has a lower frequency limit of $\sim 0.5 \mathrm{~cm}^{-1}(\lambda=20 \mathrm{~mm})$. Spectra for both coherent and normal (incoherent) emission were recorded and ratioed, thus eliminating characteristics associated with the instrument's own response. The result is a peak close to $7 \mathrm{~mm}$ wavelength (42 GHz) (see Fig. 3).

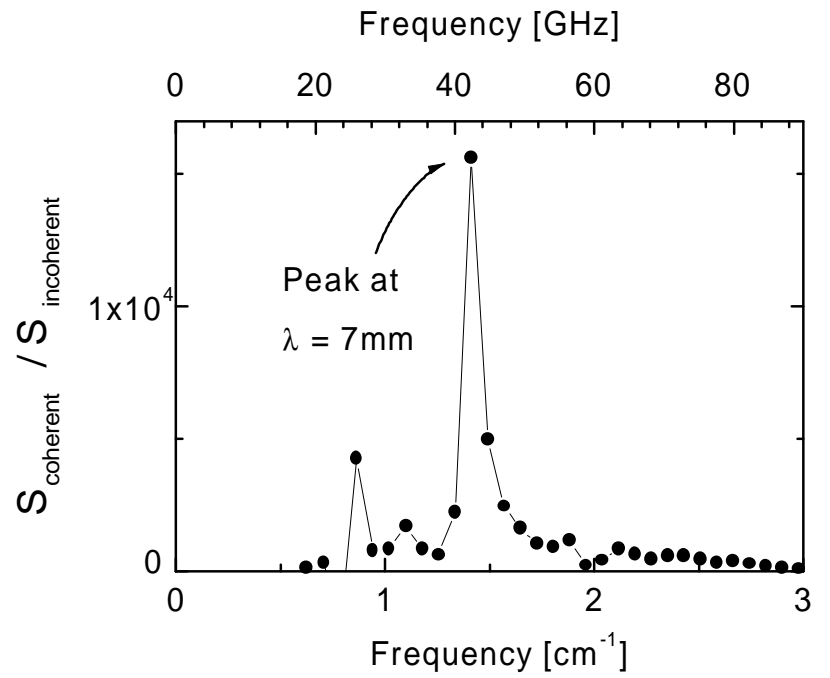

Figure 3: Power spectral content of the coherent emission, relative to the incoherent synchrotron spectrum.

\section{DISCUSSION}

\subsection{Microwave instability}

The wavelength of the emission bursts and the unusual time-structure suggest a process related to the so-called microwave instability[3]. This particular type of longitudinal instability can result when electrons interact with each other through their wake field. The wake field interaction is characterized by a complex impedance determined by the beam pipe, rf cavities, and other structures such as beam ports and bellows. The threshold condition for the microwave instability has been given by a number of authors. For unbunched (c oasting) beams, the Keil-Schnell criteria for stability[4] gives

$$
\mathrm{eI}_{\mathrm{ave}} \mathrm{Z}_{\mathrm{n}} / \mathrm{n}=2 \pi \alpha \mathrm{E} \sigma_{\mathrm{E}}^{2}
$$

where $I_{a v e}$ is the average beam current, $Z_{n}$ is the impedance for disturbance with mode number $n, \alpha$ is the momentum compaction, $\mathrm{E}$ is the electron energy and $\sigma_{\mathrm{E}}$ is the energy spread. The parameter that is most readily varied over a wide ( $>2$ orders of magnitude) range is $\alpha$. Expressed in terms of a threshold current, one finds that

$$
\mathrm{I}_{\mathrm{th}} \propto \alpha \sim \mathrm{f}_{\mathrm{s} 0}^{2}
$$

i.e., the threshold current scales linearly with the momentum compaction, or as the synchrotron frequency squared. The average current in Eq. 1 can be replaced with the peak value in order to accommodate bunched beams[5], which brings in a factor of the bunch length $\sigma_{\mathrm{L}}$. Neglecting bunch lengthening due to $\mathrm{rf}$ potential well distortion and assuming other parameters are fixed, $\sigma_{\mathrm{L}}$ depends only on the momentum compaction, and the resulting threshold condition has an 
additional factor of $\alpha^{1 / 2}$. Thus, in terms of the synchrotron frequency $\mathrm{f}_{\mathrm{s}} \sim \alpha^{1 / 2}$, we expect the threshold current to follow $\mathrm{f}_{\mathrm{s}}^{3}$ for bunched beams.

\subsection{Single bunch threshold current versus $f_{s}$}

To study the variation in the single bunch threshold current the momentum compaction of the ring was varied over a wide range $\left(-\alpha_{0} \leq \alpha \leq+2 \alpha_{0}\right)$ while holding the $\mathrm{RF}$ cavity voltage fixed. The measured threshold current behavior is shown in Fig. 4, which gives a log -log plot of the threshold current vs. synchrotron tune (in the zero current limit). The storage ring was operated under conditions of positive and negative $\alpha$. In both situations, a quadratic (as opposed to cubic) dependence of threshold current on synchrotron frequency is observed.

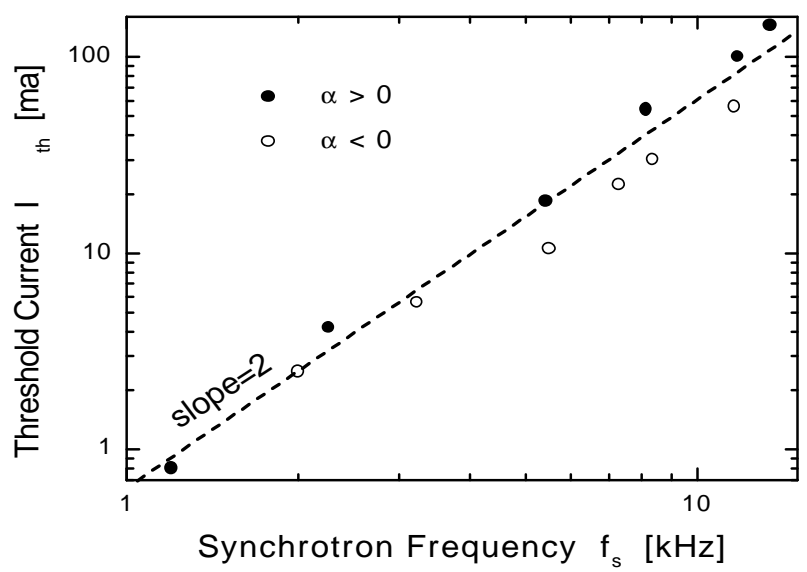

Figure 4: Measured single bunch threshold current versus synchrotron frequency for both positive (solid circles) and negative (open circles) momentum compaction. The dashed line represents the behavior $I_{t h} \sim f_{s}^{2}$.

As noted above, the rate at which the instability grows and decays is typically faster than our detector can resolve. However, we did observe a decreased growth rate for coherent bursts as $f_{s}$ (i.e., $\alpha$ ) was reduced. In particular, for $\mathrm{f}_{\mathrm{s}} \sim 1 \mathrm{kHz}$ the growth rate had a rise-time of $\sim 100 \mu \mathrm{s}$. This is shown in Figure 5, which compares the burst behavior for high $(12 \mathrm{kHz})$ and low $(1 \mathrm{kHz})$ values of $f_{s}$. While the decay rate was never resolved, we know that it must be faster than $\sim 200 \mu \mathrm{s}$, which is more than an order of magnitude shorter than the synchrotron damping time $(\sim 10 \mathrm{~ms})$. Thus, the decay is not attributed to synchrotron radiation damping. At present we do not understand what determines the time structure of the bursts. Additional studies are needed to assess the impact of potential well distortions on the threshold current.

\subsection{Beam chamber impedance}

The instability threshold depends on the beam impedance due to the storage ring vacuum chamber and any other structures subjected to the beam wake field. In practice, this impedance is dif ficult to characterize given the complexities of the rf cavities and other ring structures. But the characteristic $7 \mathrm{~mm}$ wavelength of the emission matches the corrugation period of several bellows situated around the ring chamber, and an analysis of their effective impedance at these frequencies is presently underway.

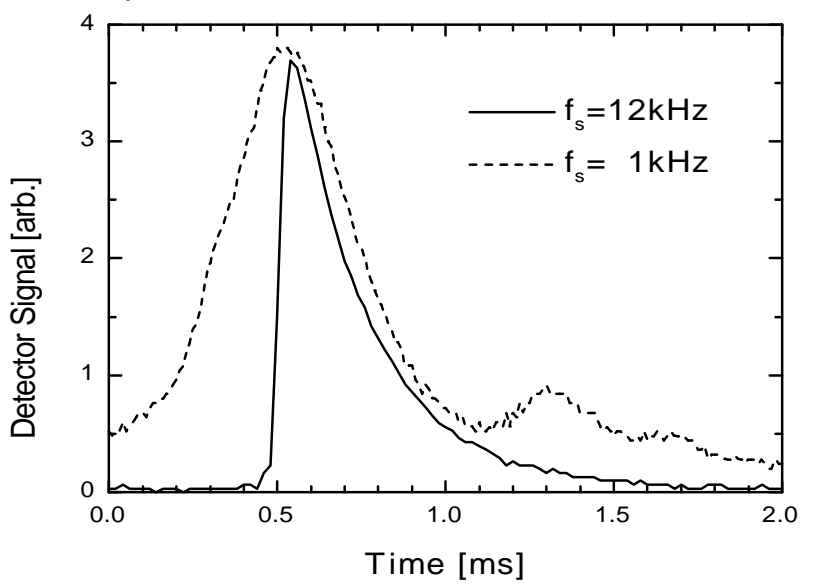

Figure 5: Coherent bursts for 2 different values of the synchrotron frequency, showing the decrease in burst risetime as the synchrotron frequency is reduced.

\section{SUMMARY}

The NSLS VUV ring produces coherent emission with a wavelength of $7 \mathrm{~mm}$. The emission appears in bursts of duration shorter than $200 \mu \mathrm{s}$, and at intervals of a few ms. Emission bursts occur only when the beam current exceeds a threshold value. The dependence of this threshold on momentum compaction is consistent with some aspects of the microwave instability, and we attribute the coherent emission to an instability -driven longitudinal density modulation. The $7 \mathrm{~mm}$ emission wavelength matches the bellows sections found at numerous locations around the ring orbit, suggesting these components produce the impedance characteristics leading to the instability. Finally, we note that bursts of coherent microwave emission have also been reported[6] by the group at SURF II (NIST).

\section{ACKNOWLEDGEMENTS}

We are grateful for assistance from G. Ramirez and the NSLS operations staff, plus beneficial conversations with J-M Wang, N. Towne, S. Krinsky and G.P. Williams.

\section{REFERENCES}

[1] S. Nodvick \& D.S. Saxon, Phys. Rev. 96, 180 (1954).

[2] G.P. Williams e t al., Phys. Rev. Lett. 62, 261 (1989); C.J. Hirschmugl et al., Phys. Rev. A44, 1316 (1991).

[3] J-M Wang \& C. Pellegrini, BNL 51236 (1979); J -M Wang, Phys. Rev. E58, 984 (1998). 
[4] E. Keil \& W. Schnell, CERN -ISR-TH-RF/69-48 (1969).

[5] D. Boussard, CERN LABII/RF/INT/75-2 (1975).

[6] A.R. Hight Walker, et. al., SPIE v.3153, p.42 (1997). 